\title{
Erratum to: Unexpected retirement from full time work after age 62: consequences for life satisfaction in older Americans
}

\author{
Philippa Clarke • Victor W. Marshall • \\ David Weir
}

Published online: 12 June 2012

(c) Springer-Verlag 2012

\section{Erratum to: Eur J Ageing \\ DOI 10.1007/s10433-012-0229-5}

In the original article, Table 3 was incorrect. The corrected table appears in this erratum.
The online version of the original article can be found under doi:10.1007/s10433-012-0229-5.

P. Clarke $(\varangle) \cdot$ D. Weir

Institute for Social Research, University of Michigan,

426 Thompson Street, Ann Arbor, MI 48104, USA

e-mail: pjclarke@umich.edu

V. W. Marshall

University of North Carolina, Chapel Hill, NC, USA
Table 3 Regression models for life satisfaction in 2008 by later life work expectations (1998-2006): U.S. Health and Retirement Study

\begin{tabular}{|c|c|c|}
\hline & $\begin{array}{l}\text { Men } \\
\beta \text { (s.e.) }\end{array}$ & $\begin{array}{l}\text { Women } \\
\beta \text { (s.e.) }\end{array}$ \\
\hline \multicolumn{3}{|c|}{ Class 1 (low expectations for working FT after age 62) } \\
\hline Intercept & $3.89(1.18)^{* * *}$ & $6.18(.66)^{* * *}$ \\
\hline Working full time in $2008^{a}$ & $-.49(.55)$ & $.32(.29)$ \\
\hline \multicolumn{3}{|c|}{ Class 2 (neutral expectations for working FT after age 62) } \\
\hline Intercept & $3.71(1.16)^{* * *}$ & $6.35(.65)^{* * *}$ \\
\hline Working full time in $2008^{a}$ & $-.39(.29)$ & $-.59(.53)$ \\
\hline \multicolumn{3}{|c|}{ Class 3 (high expectations for working FT after age 62) } \\
\hline Intercept & $3.08(1.17)^{* *}$ & $5.73(.72)^{* * *}$ \\
\hline Working full time in $2008^{a}$ & $1.40(.51)^{* *}$ & $-.15(.37)$ \\
\hline \multicolumn{3}{|l|}{ Controls } \\
\hline Age & $.20(.07)^{* *}$ & $.10(.06)$ \\
\hline White $^{\mathrm{b}}$ & $.30(.20)$ & $-.01(.23)$ \\
\hline $\begin{array}{l}\text { Less than high school } \\
\text { education }\end{array}$ & $-.25(.27)$ & $-.81(.28)^{* *}$ \\
\hline High school education $^{c}$ & $-.41(.18)^{*}$ & $-.31(.20)$ \\
\hline Number of health problems & $-.24(.06)^{* * *}$ & $-.25(.05)^{* * *}$ \\
\hline Separated/divorced ${ }^{\mathrm{d}}$ & $-.43(.30)$ & $-.88(.25)^{* * *}$ \\
\hline Widowed $^{\mathrm{d}}$ & $-.35(.45)$ & $-.65(.24)^{* *}$ \\
\hline Never married $^{\mathrm{d}}$ & $-.76(.58)$ & $-1.42(.56)^{*}$ \\
\hline $\begin{array}{l}\text { Household income (log } \\
\text { transformed) }\end{array}$ & $.15(.10)$ & $.01(.05)$ \\
\hline
\end{tabular}

FT full time, s.e. standard error

$* p<.05 ; * * p<.01 ; * * *<<.001$ (two-tailed tests)

${ }^{a}$ Reference group is not working full time in 2008

b Reference group is black or other race/ethnicity

${ }^{c}$ Reference group is college degree or higher

${ }^{\mathrm{d}}$ Reference group is married 